\title{
The Topological Spectrum of a Smooth Closed Manifold
}

\author{
JYH-YANG WU
}

\begin{abstract}
In this paper, we study the spectral geometry from the viewpoint of the theory of Hausdorff convergence. A new notion - the topological spectrum - is introduced to serve as a link between the spectral geometry and the collapsing geometry. Roughly speaking, we shall show that one can hear from the topological spectrum of a smooth manifold whether or not it is totally collapsing, non-collapsing or partially collapsing. Our main result concerning this is stated in the topological structure theorem.
\end{abstract}

1. Motivation and main results. Classically, the Hausdorff distance is a distance between two closed subsets in a fixed metric space. Around 1980, Gromov extended and gave an abstract definition of the Hausdorff distance $d_{H}$ between two metric spaces. This new version of the Hausdorff distance gives us a simple argument which can be applied to many problems in a uniform way. In particular, Hausdorff convergence along with the packing technique and comparison theorems, e.g., the Toponogov comparison theorem and the relative volume comparison theorem, provides us a powerful tool to understand some precompact classes of Riemannian manifolds. Especially, the class of Riemannian manifolds with bounded curvature and diameter.

On the other hand, the packing technique and the comparison theorem also play important roles in the spectral geometry. They are, for instance, the Cheng comparison theorem and the min-max principle. In view of this, it is natural to believe that there must be a Gromov-Hausdorff theory for the spectral geometry. The main purpose of this note is to develop this link. In this note $M$ always denotes a connected closed smooth $n$-manifold. To illustrate this link, we shall study, roughly speaking, the largest $k^{\text {th }}$ eigenvalue of the Laplace operator on $M$ that can possibly be produced. Due to the constant scaling on the Riemannian metrics, we need to normalize the metrics $g$ on $M$. Hence we shall restrict ourselves to those metrics $g$ on $M$ with the sectional curvature $|K(g)| \leq 1$. 
Definition. For a smooth closed manifold $M$, we define the topological spectrum $\operatorname{Spec}(M)=\left\{0=\lambda_{0}(M)<\lambda_{1}(M) \leq \lambda_{2}(M) \leq \lambda_{3}(M) \leq \cdots \leq\right.$ $\left.\lambda_{k}(M) \nearrow \infty\right\}$ of $M$ by

$$
\lambda_{k}(M)=\sup \left\{\lambda_{k}(M, g)|| K(g) \mid \leq 1\right\} .
$$

Remark. Note that $\lambda_{k}(M)$ 's are topological invariants of $M$ and $\lambda_{0}(M)$ is always zero, while the number $\lambda_{k}(M)$ could be infinite. Since the Laplace operator $\Delta$ acting on the $p$-forms $\Omega^{p}(M)$ also has the discrete spectrum, one may as well define and study the topological $p$-spectrum $\operatorname{Spec}_{p}(M)$ of a smooth closed manifold $M$. However in this note we shall only study the topological spectrum. From now on we shall use the conventions: $\infty \pm \alpha=\infty, \alpha \infty=\infty$, $\alpha^{\infty}=\infty$ and $\alpha^{-\infty}=0$ for any finite positive number $\alpha>1$.

The main task of this note is to illustrate that the topological spectrum $\operatorname{Spec}(M)$ indeed reveals some topological structures of the underlying manifold $M$ and it serves as the bridge between the spectral geometry and the theory of the Gromov-Hausdorff distance. First, to get some feeling of this, one can look at the following

Example 1. Let $M$ be a smooth closed $n$-manifold. Consider a Riemannian metric $g$ on $M$ with $|K(g)| \leq 1$. On the product manifold $M \times S^{1}$ of $M$ and $S^{1}=\left\{e^{i t} \mid t \in \mathbb{R}\right\}$, we construct a Riemannian metric $g_{\varepsilon}$ by $g_{\varepsilon}=g \oplus \varepsilon^{2} d t^{2}$. Thus $\left|K\left(g_{\varepsilon}\right)\right| \leq 1$. It is easy to see that

$$
\left\{\lambda_{k}\left(M \times S^{1}, g_{\varepsilon}\right) \mid k \in \mathbb{N}\right\}=\left\{\lambda_{k}(M, g)+\frac{m^{2}}{\varepsilon^{2}} \mid k \in \mathbb{N}, m \in \mathbb{Z}\right\} .
$$

Hence we have $\lim _{\varepsilon \rightarrow 0} \lambda_{k}\left(M \times S^{1}, g_{\varepsilon}\right)=\lambda_{k}(M, g)$. This implies that $\lambda_{k}(M \times$ $\left.S^{1}\right) \geq \lambda_{k}(M)$. The Weyl asymptotic formula $([\mathrm{Cl}])$ gives that $\lambda_{k}(M, g) \sim$ $c_{n} \operatorname{vol}(M, g)^{-2 / n} k^{2 / n}$ for large $k$ where $c_{n}$ is a constant depending only on the dimension $n$. Hence $\lambda_{k}\left(M \times S^{1}\right) \geq \frac{1}{2} c_{n} \operatorname{vol}(M, g)^{-2 / n} k^{2 / n}$ for large $k$. This means that the spectrum $\operatorname{Spec}\left(M \times S^{1}\right)$ has the growth of degree $\geq 2 / n$ even though the dimension of the manifold $M \times S^{1}$ is $n+1$, instead of $n$. This indicates that the topological spectrum may behave quite differently from the geometric spectrum. On the other hand, when $\varepsilon$ goes to zero, then the length of the $S^{1}$ factor in $\left(M \times S^{1}, g_{\varepsilon}\right)$ shrinks to zero. This means that $M \times S^{1}$ collapses, in the Gromov-Hausdorff topology, to $M$.

Since the $n$-torus $T^{n}$ can be obtained by the product of $S^{1}$ with itself $n$ times, this example implies that $\lambda_{k}\left(T^{n}\right)=\infty$ for all $k \geq 1$. This can also be seen from the fact that the torus $T^{n}$ admits a flat metric.

In Section 2 we shall develop some basic results about the topological spectrum. As a corollary of the well-known estimates of eigenvalues and Gromov's results on almost flat manifolds, we shall characterize all of the manifolds with $\lambda_{k}(M)=\infty$ for some $k \geq 1$ and show that they are Gromov's almost flat manifolds. 
The gap theorem. Given $k \in \mathbb{N}$ and $n \geq 2$, there exists a finite positive number $R(n, k)$ with $\lim _{k \rightarrow \infty} R(n, k) k^{-2}<\infty$ such that

(1) for an $n$-dimensional infranilmanifold $M, \lambda_{k}(M)=\infty$ for all $k \geq 1$,

(2) for an $n$-dimensional non-infranilmanifold $M, \lambda_{k}(M) \leq R(n, k)$ for all $k \geq$ 1.

Moreover, the degree of the growth of $R(n, k)$ as $k \rightarrow \infty$ is optimal for $n \neq 3$.

Recall that a manifold $M$ is called an infranilmanifold if there exists a nilpotent Lie group $N$ and a discrete subgroup $\Lambda$ of $N \propto \operatorname{Aut}(N)$ such that $M$ is diffeomorphic to $N / \Lambda$ and that $[\Lambda: N \cap \Lambda]<\infty$. This theorem tells us that we can hear from the topological spectrum whether or not $M$ is an infranilmanifold. As a corollary of this gap theorem, we shall show in Section 2 that there is an explicit universal upper bound for the Poincaré constant on the noninfranilmanifold. The numbers $R(n, k)$ can also be viewed as a universal upper bound for the topological spectrum of an $n$-dimensional non-infranilmanifold. In Section 3, Proposition 5, we shall also show, by constructing an example, that the degree of the growth of $R(n, k)$ is optimal for $n \neq 3$. A weak universal lower bound will also be discussed in Section 2 .

To study the asymptotic behavior of the topological spectrum, we introduce the following notations.

Definition. The spectral dimension, $\operatorname{dim}_{s}(M)$, of $M$ is defined to be the largest lower bound of real numbers $\alpha$ such that

$$
\lim _{t \searrow 0} t^{\alpha / 2} \sum_{k=0}^{\infty} e^{-\lambda_{k}(M) t}=0
$$

and the spectral volume $s(M)$ is defined by

$$
s(M)=\lim _{t \searrow 0}(4 \pi t)^{\operatorname{dim}_{s}(M) / 2} \sum_{k=0}^{\infty} e^{-\lambda_{k}(M) t} .
$$

Remark. Since $\lambda_{k}(M) \geq \lambda_{k}(M, g)$ for any metric $g$ on $M$ with $|K(g)| \leq 1$. This implies that $Z(M ; t):=\sum_{k=0}^{\infty} e^{-\lambda_{k}(M) t} \leq Z(M, g ; t):=\sum_{k=0}^{\infty} e^{-\lambda_{k}(M, g) t}$ for all $t>0$. Thus $Z(M ; t)$ exists for all $t>0$ and it is a decreasing function of $t$. Therefore, the definitions of the spectral dimension and the spectral volume make sense. Moreover, the Weyl asymptotic formula for $\operatorname{Spec}(M, g)$ gives $0 \leq$ $\operatorname{dim}_{s}(M) \leq \operatorname{dim}(M)$. The gap theorem also implies that for an infranilmanifold $M$ one has $\operatorname{dim}_{s}(M)=0$ and $s(M)=1$. It will be shown in Section 3 that the spectral dimension of $S^{2 n}$ is $2 n$. 
Under this terminology, the Weyl asymptotic formula still holds for the topological spectrum. Since the topological spectrum is obtained from the supremum of the geometric spectra $\operatorname{Spec}(M, g)$ of $M$ with $|K(g)| \leq 1$, we introduce a notion to describe the relation between them.

Definition. The topological spectrum $\operatorname{Spec}(M)$ of $M$ is said to be detectable if there exists a sequence of Riemannian metrics $g_{j}$ on $M$ with $\left|K\left(g_{j}\right)\right| \leq$ 1 and a positive number $\varepsilon$ such that $\liminf _{j \rightarrow \infty} \lambda_{k}\left(M, g_{j}\right) / \lambda_{k}(M) \geq \varepsilon$ for all $k \geq 1$. Otherwise, we say that the topological spectrum $\operatorname{Spec}(M)$ is undetectable. In the case that all metrics $g_{j}$ are isometric with one another, then the topological spectrum $\operatorname{Spec}(M)$ is said to be strongly detectable.

For the examples of manifolds with strongly detectable, detectable or undetectable topological spectrum, we refer to Section 3. Our main result that concerns the relation between the spectral dimension of $M$ and its topological spectrum can be described as follows.

Topological structure theorem. Let $M$ be a closed smooth n-manifold. One has:

(1) if $\operatorname{Spec}(M)$ is undetectable, then the minimal volume, $\operatorname{minvol}(M)$, is zero.

(2) $\operatorname{Spec}(M)$ is strongly detectable if and only if $\operatorname{minvol}(M)>0$.

(3) if $\operatorname{Spec}(M)$ is detectable, then there exists a compact stratified metric space $X$ with the properties:

(3.1) $\operatorname{dim}(X) \leq \operatorname{dim}_{s}(M)$;

(3.2) $X$ has a stratification $\varnothing=S_{\operatorname{dim}(X)+1}(X) \subset S_{\operatorname{dim}(X)}(X) \subset \cdots \subset S_{1}(X)$ $\subset S_{0}(X)=X$ such that $S_{k}(X)-S_{k+1}(X)$ is a smooth manifold of dimension $(\operatorname{dim}(X)-k)$;

(3.3) there is a differentiable map $f: M \rightarrow X$ such that the restriction of $f$ to $f^{-1}\left(S_{k}(X)-S_{k+1}(X)\right)$ is a fiber bundle whose fiber is diffeomorphic to an infranilmanifold.

(3.4) $X$ is isometric to the quotient space $N / O(n)$ of a smooth manifold $N$ with a $C^{1, \alpha}$-Riemannian metric by a smooth and isometric action of the orthogonal group $O(n)$. Moreover, the map $f: M \rightarrow X$ lifts to a map $\tilde{f}$ from a principle bundle $P M$ of $M$ with the structure group $O(n)$ into $N$ such that $\tilde{f}$ is a fiber bundle with an infranilmanifold fiber.

Remark. The minimal volume, $\operatorname{minvol}(M)$ of a smooth manifold $M$ is defined to be $\inf \{\operatorname{vol}(M, g)|| K(g) \mid \leq 1\}$. It is now well known that the minimal volume of $M$ is bounded below by Gromov's simplicial volume of $M$. For discussions about the minimal volume, we refer to Gromov [G1]. Consider the topological spectrum of a manifold which is not strongly detectable and which is detectable. Then the volumes of any detectable sequence necessarily converge to 
zero. To prove this structural result, we shall establish in Section 3 the totally collapsing theorem, the non-collapsing theorem and the direct and inverse collapsing theorems and then appeal to a compactification result of Fukaya. Then one can see from these results that there is a strong link between the spectral geometry and the Gromov-Hausdorff theory.

Section 2. The upper and lower bounds for the topological spectrum. Before we discuss the relation between the spectral geometry and the collapsing geometry, we establish some basic properties about the topological spectrum. First, let us investigate some 2-dimensional manifolds other than a 2-torus.

Example 2. A result of Hersch $([\mathrm{H}])$ states that every Riemannian metric $g$ on the 2 -sphere, $S^{2}$, has

$$
\lambda_{1}\left(S^{2}, g\right) \operatorname{vol}\left(S^{2}, g\right) \leq 8 \pi
$$

where $\operatorname{vol}\left(S^{2}, g\right)$ denotes the volume of $\left(S^{2}, g\right)$ and the equality holds only when $g$ has positive constant curvature. On the other hand, the Gauss-Bonnet theorem implies that

$$
2 \pi \chi\left(S^{2}\right)=\int_{S^{2}} K(g) d \operatorname{vol}_{g} \leq \operatorname{vol}\left(S^{2}, g\right)
$$

for any metric $g$ on $S^{2}$ with $|K(g)| \leq 1$. These two inequalities together show that $\lambda_{1}\left(S^{2}\right)=2$ and the value 2 is obtained by the canonical metric $g_{\text {can }}$ on $S^{2}$ with the constant curvature +1 . Using a result of Li and Yau ([LY]), a similar argument also yields that $\lambda_{1}\left(\mathbb{R} P^{2}\right)=6$.

Example 3. Yang and Yau proved in $[\mathrm{YY}]$ that for any compact orientable two-manifold $M_{g}$ with genus $g(M)$ one has

$$
\lambda_{1}\left(M_{g}, h\right) \operatorname{vol}\left(M_{g}, h\right) \leq 8 \pi(g(M)+1)
$$

for any Riemannian metric $h$ on $M_{g}$. Again the Gauss-Bonnet theorem gives that for $g(M) \geq 2$,

$$
\operatorname{vol}\left(M_{g}, h\right) \geq 2 \pi\left|\chi\left(M_{g}\right)\right|=4 \pi(g(M)-1)
$$

for any metric $h$ on $M_{g}$ with $|K(h)| \leq 1$, and the equality is obtained by the hyperbolic metrics on $M_{g}$. Therefore, one has $\lambda_{1}\left(M_{g}\right) \leq 2(g(M)+1) /(g(M)-1)$. However this estimate is far from optimal. Indeed, for any $k \geq 1$ and $\varepsilon>0$, there is an integer $g(k, \varepsilon)$ such that $\lambda_{k}\left(M_{g}\right) \leq \frac{1}{4}+\varepsilon$ as long as $g(M) \geq g(k, \varepsilon)$; see Proposition 2 below. 
To prove the gap theorem for the topological spectrum we first define the minimal diameter, $d(M)$, of a smooth closed manifold $M$ by

$$
d(M)=\inf \{\operatorname{diam}(M, g)|| K(g) \mid \leq 1\}
$$

where $\operatorname{diam}(M, g)$ denotes the diameter of $(M, g)$.

Under the notion of the minimal diameter, a famous theorem of Gromov ([G2], $[\mathrm{BK}])$, refined by $\operatorname{Ruh}([\mathrm{R}])$, can be stated as:

Theorem 1. A smooth closed n-manifold $M$ has $d(M) \leq d_{n}:=$ $\exp \left(-\exp \left(\exp n^{2}\right)\right)$ if and only if $M$ is an infranilmanifold and $d(M)=0$.

The proof of the first part of our gap theorem for the topological spectrum is based on a combination of some results of Cheng, Gromov, Berard, Besson and Gallot concerning the upper and lower bounds on the eigenvalues of the Laplace operator.

Theorem 2 (Cheng [Ch], $[\mathrm{Cl}])$. Let $(M, g)$ be a Riemannian n-manifold with $K(g) \geq-1$. Then one has $\lambda_{k}(M, g) \leq \lambda(\operatorname{diam}(M, g) / 2 k)$ for all $k \geq 1$ where $\lambda(r)$ denotes the lowest Dirichlet eigenvalue of the Laplace operator on the $r$-ball, $B(r)$, in the simply connected space $n$-form $M_{-1}^{n}$ with the constant curvature -1

Theorem 3 (Gromov [G3], Berard-Besson-Gallot [BBG]). Let $(M, g)$ be a Riemannian $n$-manifold with $K(g) \operatorname{diam}(M, g)^{2} \geq-\alpha^{2}, \alpha>0$. Then there exists a positive number $c(n, \alpha)$ depending on $n$ and $\alpha$ such that

$$
\lambda_{k}(M, g) \geq c(n, \alpha) k^{2 / n} \operatorname{diam}(M, g)^{-2}
$$

for all $k \geq 1$.

Now we are in a position to investigate the manifolds $M$ with $\lambda_{k}(M)=\infty$ for some $k \geq 1$.

Proposition 1. Let $M$ be a smooth closed $n$-manifold. Then the following statements are equivalent:

(1) $\lambda_{k}(M)=\infty$ for all $k \geq 1$;

(2) $\lambda_{k}(M)=\infty$ for some $k \geq 1$;

(3) $d(M)=0$. 
Proof. (1) $\Rightarrow(2)$ is obvious. (2) $\Rightarrow(3)$ : Since $\lambda_{k}(M)=\infty$ for some $k \geq 1$, there is a sequence of Riemannian metrics $g_{j}$ on $M$ with $\left|K\left(g_{j}\right)\right| \leq 1$ and $\lambda_{k}\left(M, g_{j}\right) \rightarrow \infty$ as $j \rightarrow \infty$. Theorem 2 then gives that $\lambda_{k}\left(M, g_{j}\right) \leq$ $\lambda\left(\operatorname{diam}\left(M, g_{j}\right) / 2 k\right)$ for all $j$. Hence $\lim _{j \rightarrow \infty} \lambda\left(\operatorname{diam}\left(M, g_{j}\right) / 2 k\right)=\infty$. This implies that $\lim _{j \rightarrow \infty} \operatorname{diam}\left(M, g_{j}\right)=0$. Thus $d(M)=0$. Next we show $(3) \Rightarrow$ (1). Since $d(M)=0$, there exists a sequence of Riemannian metrics $g_{j}$ on $M$ with $|K(g)| \leq 1$ and $\operatorname{diam}\left(M, g_{j}\right) \rightarrow 0$ as $j \rightarrow \infty$. We can assume that $\operatorname{diam}\left(M, g_{j}\right) \leq 1$ for all $j$. Then Theorem 3 implies for $\alpha=1$ that $\lambda_{k}\left(M, g_{j}\right) \geq$ $c(n, \alpha) k^{2 / n} \operatorname{diam}\left(M, g_{j}\right)^{-2}$ for all $k \geq 1$ and $j$. This gives $\lambda_{k}(M) \geq$ $\lim _{j \rightarrow \infty} \lambda_{k}\left(M, g_{j}\right)=\infty$. Thus $\lambda_{k}(M)=\infty$ for all $k \geq 1$. This completes the proof of Proposition 1.

Combining this proposition with Theorem 1, we can now obtain the gap theorem mentioned in the introduction.

Proof of the gap theorem. Since an infranilmanifold $M$ has $d(M)=0$, Proposition 1 implies that $\lambda_{k}(M)=\infty$ for all $k \geq 1$. Theorem 1 gives that an $n$-dimensional non-infranilmanifold $M$ must have $d(M)>d_{n}$. Then Theorem 2 implies that $\lambda_{k}(M, g) \leq \lambda(\operatorname{diam}(M, g) / 2 k) \leq \lambda\left(d_{n} / 2 k\right)$ for all $k \geq 1$ and any metric $g$ on $M$ with $|K(g)| \leq 1$. Set $R(n, k)=\lambda\left(d_{n} / 2 k\right)$. Then we have $\lambda_{k}(M) \leq R(n, k)$ for all $k \geq 1$. Since $\lim _{\varepsilon \rightarrow 0} \varepsilon^{2} \lambda(\varepsilon)=c_{n}$ where $c_{n}$ is a constant depending only on the dimension $n$. Thus we have $\lim _{k \rightarrow \infty} R(n, k) k^{-2}=$ $\lim _{k \rightarrow \infty} \lambda\left(d_{n} / 2 k\right) k^{-2}=4 c_{n} / d_{n}^{2}<\infty$ and the first part of the gap theorem holds. For the second part about the optimality of the degree of the growth of $R(n, k)$, we postpone the proof to Section 3, Proposition 5 .

The Poincare inequality states that for any closed Riemannian $n$-manifold $(M, g)$ there exists a constant $c>0$ such that

$$
\int_{M}|\nabla f|^{2} d \operatorname{vol}_{g} \geq c \int_{M} f^{2} d \operatorname{vol}_{g}
$$

for all $f \in C^{\infty}(M)$ with $\int_{M} f d \operatorname{vol}_{g}=0$. We define the Poincaré constant $p(M, g)$ to be the largest positive constant $c$ with the property $(+)$. It is well-known, by the min-max principle, that the constant $p(M, g)$ is the smallest nonzero eigenvalue $\lambda_{1}(M, g)$ of the Laplace operator $\Delta$ on $(M, g)$. Therefore, the gap theorem implies that there is an explicit universal upper bound for the Poincaré constant of a non-infranilmanifold.

Theorem 4. For $n \geq 2$, there exists a constant $p(n) \leq \exp \left(5 \exp \left(\exp n^{2}\right)\right)$ such that $p(M, g) \leq p(n)$ for any Riemannian metric $g$ on an $n$-dimensional non-infranilmanifold $M$ with $|K(g)| \leq 1$. 
Proof. The gap theorem implies that $p(M, g)=\lambda_{1}(M, g) \leq \lambda_{1}(M) \leq$ $R(n, 1)$ for any Riemannian metric $g$ on an $n$-dimensional non-infranilmanifold $M$ with $|K(g)| \leq 1$. To complete the proof, we only need to obtain the explicit upper bound for $R(n, 1)$. From the proof of the gap theorem, one has $R(n, 1)=\lambda\left(d_{n} / 2\right)$ with $d_{n}=\exp \left(-\exp \left(\exp n^{2}\right)\right)$. On the other hand, we have $([\mathrm{Cl}]$, p. 49) an upper bound for $\lambda(r), r>0$. Namely,

$$
\begin{aligned}
\sqrt{\lambda(r)} \leq(n-1) & \frac{\operatorname{coth}(r / 2)-1}{2} \\
& +\left[\frac{(n-1)^{2}}{4}+\frac{4 \pi^{2}}{r^{2}}+(n-1)^{2}\left(\frac{\operatorname{coth}(r / 2)-1}{2}\right)^{2}\right]^{1 / 2}
\end{aligned}
$$

for all $r>0$. An easy computation then gives the rough estimate:

$$
\lambda(r) \leq \frac{16 n^{4} \pi^{4}}{r^{4}}
$$

for $0<r<\frac{1}{10}$. Finally, we have

$$
p(M, g) \leq \lambda\left(\frac{d_{n}}{2}\right) \leq \frac{256 n^{4} \pi^{4}}{\exp \left(-4 \exp \left(\exp n^{2}\right)\right)} \leq \exp \left(5 \exp \left(\exp n^{2}\right)\right) .
$$

The constants $R(n, k)$ in the gap theorem can also be viewed as a universal upper bound for $\lambda_{k}(M)$ of an $n$-dimensional non-infranilmanifold $M$. It is natural to ask whether there is a universal lower bound $r(n, k)$ for $\lambda_{k}(M)$ of an $n$-manifold $M$ with $r(n, k) \rightarrow \infty$ as $k \rightarrow \infty$. In fact, this is not possible and it can be seen from the following:

Proposition 2. Given $k \in \mathbb{N}$ and $\varepsilon>0$, there exists a smooth closed $n$ manifold $M_{\varepsilon}$ with $\lambda_{k}\left(M_{\varepsilon}\right) \leq(n-1)^{2} / 4+\varepsilon$.

Proof. Take a sequence of smooth closed $n$-manifold $M_{j}$ with the total Betti number $b\left(M_{j}\right):=\sum_{i=0}^{n} \operatorname{dim} \mathbb{H}^{i}\left(M_{j} ; \mathbb{R}\right) \rightarrow \infty$ as $j \rightarrow \infty$. For example, take $M_{j}=$ $N_{j} \times S^{n-2}$ with $N_{j}$ the orientable two-surface with genus $g\left(N_{j}\right)=j$. A result of Gromov ([G4]) on the total Betti number asserts that there exists an increasing continuous function $f_{n}: \mathbb{R}^{+} \cup\{0\} \rightarrow \mathbb{R}^{+}$such that $b(M) \leq f_{n}(\operatorname{diam}(M, g))$ for any Riemannian metric $g$ on $n$-manifold $M$ with $K(g) \geq-1$. In particular, $b\left(M_{j}\right) \leq f_{n}\left(d\left(M_{j}\right)\right)$. Hence we have $\lim _{j \rightarrow \infty} d\left(M_{j}\right)=\infty$. Theorem 2 also implies that $\lambda_{k}\left(M_{j}\right) \leq \lambda\left(d\left(M_{j}\right) / 2 k\right)$. According to a result of McKean ([M], $[\mathrm{Cl}])$, one has $\lim _{r \rightarrow \infty} \lambda(r)=(n-1)^{2} / 4$. Therfeore, we obtain $\lim _{j \rightarrow \infty} \lambda_{k}\left(M_{j}\right) \leq$ $(n-1)^{2} / 4$ and Proposition 2 follows. 
Remark. Proposition 2 is, in spirit, similar to a theorem of Buser ([Bu]) about the Riemannian surface. It states that given positive integers $g$ and $k$ and any $\varepsilon>0$, there exists a hyperbolic metric $h$ on the two-surface $M_{g}$ of genus $g$ satisfying $\lambda_{k}\left(M_{g}, h\right) \leq \frac{1}{4}+\varepsilon$.

Although Proposition 2 says that for any $k \geq 1$ there exists an $n$-manifold $M_{k}$ with $\lambda_{k}\left(M_{k}\right) \leq n^{2} / 4$, for a fixed $n$-manifold $M \lambda_{k}(M)$ still has the growth of degree at least $2 / n$. Thus $\lambda_{k}(M)$ will eventually be greater than $n^{2} / 4$ for some $k_{0}$ and all $k \geq k_{0}$. For some manifolds the integers $k_{0}$ are small but for others they may be large. Hence it is a natural guess that for those manifolds with small $k_{0}, \lambda_{k}(M)$ will grow faster. This phenomenon is illustrated by the following weak universal lower bound for the topological spectrum.

A lower bound theorem. For any $n \geq 2$, there exists a constant $m_{n}$ such that if $M$ is an $n$-manifold with $\lambda_{k_{0}}(M)>n^{2} / 4$, then

$$
\lambda_{k}(M) \geq \frac{k^{2 / n}}{e^{k_{0} m_{n}}}
$$

for all $k \geq k_{0}$.

To prove this theorem, we shall use a result of Berard, Besson and Gallot.

Theorem 5 (Berard-Besson-Gallot $[\mathrm{BBG}])$. Let $(M, g)$ be a Riemannian $n$-manifold with $K(g) \operatorname{diam}(M, g)^{2} \geq-\alpha^{2}, \alpha>0$. Then

$$
Z(M, g ; t) \leq Z\left(S^{n}, \operatorname{can} ; \frac{t}{r^{2}}\right)
$$

where $r=\operatorname{diam}(M, g) / \alpha \tilde{c}(\alpha)$ and $\tilde{c}(\alpha)$ is the unique positive root of the equation for $x$ :

$$
x \int_{0}^{\alpha}(\cosh t+x \sinh t)^{n-1} d t=\frac{\operatorname{vol}\left(S^{n}, \operatorname{can}\right)}{\operatorname{vol}\left(S^{n-1}, \operatorname{can}\right)} .
$$

Proof of a lower bound theorem. By the gap theorem, this theorem is true for any infranilmanifold $M$ since $\lambda_{k}(M)=\infty$ for all $k \geq 1$. Thus we can assume that $M$ is an $n$-dimensional non-infranilmanifold. Hence one has $d(M)>d_{n}$. For any metric $g$ on $M$ with $|K(g)| \leq 1$, Theorem 5 implies that $Z(M, g ; t) \leq Z\left(S^{n}, \operatorname{can}, t / r^{2}\right)$ with $r=1 / \tilde{c}(\operatorname{diam}(M, g))$. Thus we obtain

$$
Z(M ; t) \leq Z(M, g ; t) \leq Z\left(S^{n}, \operatorname{can} ; \tilde{c}(\operatorname{diam}(M, g)) t\right) .
$$

for all $t>0$. This then gives

$$
Z(M ; t) \leq Z\left(S^{n}, \operatorname{can} ; \tilde{c}(d(M)) t\right) .
$$


The Minakshisundaram-Pleijel asymptotic expansion ([BGM]) implies that there exists a constant $c_{1}(n)$ such that

$$
Z\left(S^{n}, \operatorname{can} ; t\right) \leq c_{1}(n) t^{-n / 2}
$$

for any positive $t \leq \tilde{c}\left(d_{n}\right)$. From the definition of the number $\tilde{c}(\alpha)$, one can see easily that $\tilde{c}(\alpha)$ is a decreasing function of $\alpha$. Hence $\tilde{c}\left(d_{n}\right) \geq \tilde{c}(d(M))$. Inequalities (2) and (3) give that for any positive $t \leq 1$,

$$
Z(M ; t) \leq c_{1}(n) \tilde{c}(d(M))^{-n} t^{-n / 2} .
$$

Since $1-\lambda_{j}(M) / \lambda_{k}(M) \geq 0$ for any $j \leq k$, we have

$$
k \leq \sum_{j=1}^{k} e^{1-\lambda_{j}(M) / \lambda_{k}(M)} \leq e Z\left(M ; \frac{1}{\lambda_{k}(M)}\right) .
$$

Since $\lambda_{k_{0}}(M)>n^{2} / 4 \geq 1$, inequalities (4) and (5) imply that for $k \geq k_{0}$,

$$
k \leq e c_{1}(n) \tilde{c}(d(M))^{-n} \lambda_{k}(M)^{n / 2} .
$$

Simplify this to yield

$$
\lambda_{k}(M) \geq \tilde{c}(d(M))^{2}\left(\frac{k}{e c_{1}(n)}\right)^{n / 2}
$$

as long as $k \geq k_{0}$.

To complete the proof we need to obtain a lower bound for $\tilde{c}(d(M))$. Since $\lambda_{k_{0}}(M)>n^{2} / 4$, there exists a Riemannian metric $g$ on $M$ with $|K(g)| \leq 1$ and $\lambda_{k_{0}}(M, g) \geq n^{2} / 4$. According to Theorem 2 , we have $n^{2} / 4 \leq \lambda\left(\operatorname{diam}(M, g) / 2 k_{0}\right)$.

Since $\lambda(r)$ is also a decreasing function of $r$ with $\lim _{r \rightarrow \infty} \lambda(r)=(n-1)^{2} / 4$, there exists a unique positive constant $\alpha_{n}$ such that $\lambda\left(\alpha_{n}\right)=n^{2} / 4$. Thus one has $\operatorname{diam}(M, g) / 2 k_{0} \leq \alpha_{n}$. This implies $d_{n}<d(M) \leq 2 k_{0} \alpha_{n}$.

Since $\tilde{c}(\alpha)$ is a decreasing function of $\alpha$, we have $\tilde{c}(d(M)) \geq \tilde{c}\left(2 k_{0} \alpha_{n}\right)$. This along with (7) gives

$$
\lambda_{k}(M) \geq \tilde{c}\left(2 k_{0} \alpha_{n}\right)^{2}\left(\frac{k}{e c_{1}(n)}\right)^{n / 2} .
$$

for all $k \geq k_{0}$. Again from the definition of $\tilde{c}(\alpha)$, it is easy to verify that

$$
\tilde{c}\left(2 k_{0} \alpha_{n}\right) \geq \frac{\operatorname{vol}\left(S^{n}, \operatorname{can}\right)}{\operatorname{vol}\left(S^{n-1}, \operatorname{can}\right) e^{3(n-1) k_{0} \alpha_{n}}} .
$$

From (8) and (9), we can conclude that there exists a constant $m_{n}$ such that

$$
\lambda_{k}(M) \geq \frac{k^{n / 2}}{e^{k_{0} m_{n}}} .
$$

for all $k \geq k_{0}$ and this completes the proof of Theorem 5 . 
Remark. From the statement of the lower bound theorem, one may expect to have a universal lower bound of the form $\lambda_{k}(M) \geq \lambda_{k_{0}}(M)+a\left(n, k, k_{0}\right)$ for all $k \geq k_{0}$ with $a\left(n, k, k_{0}\right)>0$. This is not possible since $M$ may have a repeated eigenvalue $\lambda_{k}(M)=\lambda_{k+1}(M)$.

Section 3. Topological spectrum and collapsing phenomenon. In this section we try to study the spectral geometry from the viewpoint of collapsing geometry. This is also part of the reason why we introduce the new notion of the topological spectrum. We shall show that one can hear from the topological spectrum $\operatorname{Spec}(M)$ whether the manifold $M$ is non-collapsong, partially collapsing or totally collapsing. To illustrate this, we need a definition to descibe the collapsing dimension of an $n$-manifold $M$.

We shall say that a compact metric space $(X, d)$ is a limit space of $M$ if there exists a sequence of Riemannian metrics $g_{j}$ on $M$ with $\left|K\left(g_{j}\right)\right| \leq 1$ such that $(X, d)$ is the limit space of $\left(M, d_{j}\right)$ in the Hausdorff topology where $d_{j}$ is the induced metric on $M$ by $g_{j}$. That is, $\lim _{j \rightarrow \infty} d_{H}\left(\left(M, d_{j}\right),(X, d)\right)=$ 0 in the Hausdorff metric $d_{H}$. Note that the metric space $X$ may not be a Riemannian manifold. We refer to Fukaya [F1], Gromov [G5] and Wu [W1] for the discussions about the Hausdorff distance between two compact metric spaces. In the case that $(X, d)$ is a compact smooth manifold with the metric $d$ induced by a Riemannian metric $g$ on $X$, we shall say that $M$ collapses to $X$. Under this terminology, the collapsing dimension, $\operatorname{dim}_{c}(M)$, is defined by

$$
\operatorname{dim}_{c}(M)=\min \{\operatorname{dim}(X, d) \mid(X, d) \text { is a compact limit space of } M\} .
$$

Note that in the definition of $\operatorname{dim}_{c}(M)$ the compact space $X$ is not required to be smooth. One always has $0 \leq \operatorname{dim}_{c}(M) \leq \operatorname{dim}(M)$. A manifold $M$ is said to be totally collapsing if $\operatorname{dim}_{c}(M)=0$; non-collapsing if $\operatorname{dim}_{c}(M)=\operatorname{dim}(M)$ or partially collapsing if $0<\operatorname{dim}_{c}(M)<\operatorname{dim}(M)$.

For an $n$-manifold $M$ with $\operatorname{minvol}(M)>0$ the Gromov compactness theorem ([G5]) implies that any compact limit metric space $(X, d)$ of $M$ is $n$-dimensional. Thus in this case $M$ has $\operatorname{dim}_{c}(M)=n$ and is non-collapsing. On the other hand, it is easy to see that if $d(M)=0$, then $M$ is totally collapsing.

Example 4. $\operatorname{dim}_{c}\left(S^{3}\right)=1$. To see this, we consider the group $S O(4)$ acting on the standard 3 -sphere, $\left(S^{3}, g_{\text {can }}\right)$ by isometries. Take a dense subgroup $\mathbb{R}$ in the maximal torus $T^{2}$ of $S O(4)$. For any positive $\varepsilon<<1$ and a tangent vector $v$ of $S^{3}$, we put

$$
g_{\varepsilon}=\left\{\begin{aligned}
\varepsilon g_{\mathrm{can}}(v, v) & \text { if } v \text { is tangent to an } \mathbb{R} \text { orbit } \\
g_{\mathrm{can}}(v, v) & \text { if } v \text { is perpendicular to an } \mathbb{R} \text { orbit }
\end{aligned}\right.
$$

Then we have $\left|K\left(g_{\varepsilon}\right)\right| \leq 1$ and $\lim _{\varepsilon \rightarrow 0}\left(S^{3}, g_{\varepsilon}\right)=\left([0, \pi / 2], d t^{2}\right)$. This shows

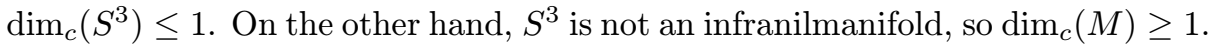
Thus one has $\operatorname{dim}_{c}(M)=1$. 
In the context of collapsing geometry two questions arise naturally:

(1) If $M$ collapses to $N$ and $N$ collapses to $X$, will $M$ collapse to $X$ ?

(2) If $M$ collapses to $N$, is it true that $\operatorname{dim}_{c}(M) \leq \operatorname{dim}_{c}(N)$ ?

The answer to both of these questions is no. This can be seen from the following example.

Example 5. Take a solv-manifold $M$ (compact quotient of a solvable Lie group) which is not an infranilmanifold. Hence $\operatorname{dim}_{c}(M) \geq 1$. It is well known (cf. [Fu1] [P]) that $M$ collapses to a $k$-torus $T^{k}$. It is clear that $T^{k}$ collapses to a point. Thus $\operatorname{dim}_{c}\left(T^{k}\right)=0$. Moreover, $M$ can not collapse to a point since it is not an infranilmanifold.

We now return to the discussion about the topological spectrum. First of all let us develop a Weyl-type asymptotic formula.

The Weyl-type asymptotic formula. For a smooth closed manifold $M$ we have:

(1) if $\beta>\operatorname{dim}_{s}(M)$, then $\lambda_{k}(M) \geq c_{\beta} k^{2 / \beta}$ for all $k \geq 1$ where $c_{\beta}$ is a finite positive number $c_{\beta}$ depending on $M$ and $\beta$;

(2) if $0<\beta<\operatorname{dim}_{s}(M)$, then $\lambda_{k}(M) \leq c_{\beta} k^{2 / \beta}$ all $k \geq 1$ where $c_{\beta}$ is a finite positive number $c_{\beta}$ depending on $M$ and $\beta$;

(3) if $s(M)$ is a finite positive number, then $\lambda_{k}(M) \sim c(m) s(M)^{-2 / m} k^{2 / m}$ for large $k$ where $m=\operatorname{dim}_{s}(M)$ and the constant $c(m)$ depends only on the spectrum dimension $\operatorname{dim}_{s}(M)$ of $M$.

Proof. This result is basically well-known. The cases (1), (2) and (3) can be verified in a similar way by using the Karamata's Tauberian theorem $([\mathrm{Fe}])$. Hence we shall briefly discuss the proof of (1). We use the notation $f(t) \stackrel{t \rightarrow \alpha}{\leq} g(t)$, $\alpha=0^{+}$or $\infty$ when $\lim _{t \rightarrow \alpha} f(t) / g(t)<\infty$. Since $\beta>\operatorname{dim}_{s}(M)$, we have

$$
\sum_{k=0}^{\infty} e^{-\lambda_{k}(M) t} \stackrel{t \rightarrow 0}{\leq} t^{-\beta / 2}
$$

Now $Z(M ; t)=\sum_{k=0}^{\infty} e^{-\lambda_{k}(M) t}$ can be viewed as the Laplace transform of the measure $\mu$ which assigns the weight 1 to the point $\lambda_{k}(M)$. Let $U$ be its distribution function defined for all $x \geq 0$. That is,

$$
U(x)=\sum_{\lambda_{k}(M) \leq x} 1 .
$$


Since $\beta / 2 \geq 0$, we can apply Karamata's Tauberian theorem to obtain

$$
U(x) \stackrel{x \rightarrow \infty}{\leq} x^{\beta / 2} .
$$

This then implies that for $x=\lambda_{k}(M)$,

$$
k \stackrel{k \rightarrow \infty}{\leq} \lambda_{k}(M)^{\beta / 2} .
$$

One can conclude from (4) that there exists a positive finite constant $c_{\beta}$ such that for all $k \geq 1$ one has

$$
\lambda_{k}(M) \geq c_{\beta} k^{2 / \beta}
$$

and this completes the proof of (1).

As an application of this formula, we show:

Proposition 3. For a non-infranilmanifold $M$ one has $\operatorname{dim}_{s}(M) \geq 1$.

Proof. The gap theorem says that a non-infranilmanifold $M$ of dimension $n$ has $\lambda_{k}(M) \leq R(n, k)$ for all $k \geq 1$. Since $\lim _{k \rightarrow \infty} R(n, k) k^{-2}<\infty$, there exists a constant $c_{n}$ such that $\lambda_{k}(M) \leq c_{n} k^{2}$ for all $k \geq 1$. If $\operatorname{dim}_{s}(M)<1$, the Weyl-type asymptotic formula implies for any $\beta$ with $\operatorname{dim}_{s}(M)<\beta<1$ that there exists a positive constant $c_{\beta}$ such that $\lambda_{k}(M) \geq c_{\beta} k^{2 / \beta}$ for all $k \geq 1$. This contradicts the fact $\lambda_{k}(M) \leq c_{n} k^{2 / \beta}$ for all $k \geq 1$. Hence $\operatorname{dim}_{s}(M) \geq 1$.

This proposition along with Example 5 shows that if $M$ collapses to $N$, then it is not necessary that $\operatorname{dim}_{s}(M) \leq \operatorname{dim}_{s}(N)$. The gap theorem and this proposition also imply:

The totally collapsing theorem. For a closed smooth manifold $M$, one has that $\operatorname{dim}_{c}(M)=0 \Leftrightarrow d(M)=0 \Leftrightarrow \lambda_{k}(M)=\infty$ for some $k \geq 1 \Leftrightarrow$ $\operatorname{dim}_{s}(M)=0 \Leftrightarrow M$ is an infranilmanifold.

Now we start to investigate the topological spectrum of a non-collapsing manifold.

Proposition 4. If $\operatorname{Spec}(M)=\operatorname{Spec}(M, h)$ for some Riemannian metric $h$ on $M$ with $|K(h)| \leq 1$, we have $\operatorname{minvol}(M)=\operatorname{vol}(M, h)$.

Proof. From the fact $\operatorname{Spec}(M)=\operatorname{Spec}(M, h)$, we obtain $Z(M ; t)=Z(M, h ; t)$ for all $t>0$. For any Riemannian metric $g$ on $M$ with $|K(g)| \leq 1$ one has $Z(M ; t) \leq Z(M, g ; t)$ for all $t>0$. Hence $Z(M, h ; t) \leq Z(M, g ; t)$ for all $t>0$. The Minakshisundaram-Pleijel asymptotic expansion implies for the metrics $h$ and $g$ to obtain $\operatorname{vol}(M, h) \leq \operatorname{vol}(M, g)$. Thus we have $\operatorname{vol}(M, h)=$ $\operatorname{minvol}(M)$. 
Remark. The converse of this proposition is not true. Namely, if $\operatorname{minvol}(M)=\operatorname{vol}(M, h)$ for some Riemannian metric $h$ on $M$ with $|K(h)| \leq 1$, it is not necessary that $\lambda_{k}(M)=\lambda_{k}(M, h)$. To see this we note that any hyperbolic metric $h$ on an orientable two-surface $M_{g}$ with genus $g \geq 2$ has $\operatorname{vol}(M, h)=\operatorname{minvol}(M)=4 \pi(g-1)$. A result of Buser $([\mathrm{B}])$ asserts that given any $\varepsilon>0$ and integer $g \geq 2$, there is a hyperbolic metric $h$ on $M_{g}$ such that $\lambda_{2 g-3}(M, h)<\varepsilon$.

Next we shall show that one can hear from the topological spectrum $\operatorname{Spec}(M)$ whether or not the manifold $M$ is collapsing. More precisely, we have:

The non-collapsing theorem. For an n-manifold $M$ the following statements are equivalent:

(1) $\operatorname{Spec}(M)$ is strongly detectable by all Riemannian metrics $g$ on $M$ with $|K(g)| \leq 1$;

(2) $\operatorname{Spec}(M)$ is strongly detectable by some Riemannian metric $g$ on $M$ with $|K(g)| \leq 1$

(3) $\operatorname{minvol}(M)>0$;

(4) $\limsup _{k \rightarrow \infty} \lambda_{k}(M) k^{2 / n}<\infty$, and

(5) $\operatorname{dim}_{s}(M)=n$ and $s(M)>0$.

To prove this theorem we introduce the concept about the essential packing of a smooth $n$-manifold $M$. Let $g$ be a Riemannian metric on $M$. Given any $\varepsilon>0$ let $\left\{x_{i}\right\}_{i=1}^{P(M, g ; \varepsilon)}$ denote a maximal set of points in $M$ such that the $\varepsilon$-balls $B\left(x_{i}, \varepsilon\right)$ in $(M, g)$ are pair-wise disjoint and their union $\bigcup_{i=1}^{P(M, g ; \varepsilon)} B\left(x_{i}, 2 \varepsilon\right)=M$. We shall call $P(M, g ; \varepsilon)$ the packing function of $(M, g)$. Note that this definition of the packing function also makes sense for compact metric spaces. The essential packing function $P(M ; \varepsilon)$ of $M$ is then defined by

$$
P(M ; \varepsilon)=\min \{P(M, g ; \varepsilon)|| K(g) \mid \leq 1\} .
$$

From the result of Gromov's almost flat manifolds, we have $d(M)=0 \Leftrightarrow$ $P(M ; \varepsilon)=1$ for all $\varepsilon>0$. For the packing function $P(M, g ; \varepsilon)$ one also has a different version of the Cheng comparison theorem.

Theorem 2A $([\mathrm{Be}])$. Let $(M, g)$ be a Riemannian $n$-amnifold with $K(g) \geq$ -1 . Then one has $\lambda_{P(M, g ; \varepsilon)}(M, g) \leq \lambda(\varepsilon)$ for any $\varepsilon>0$. 
Proof of the non-collapsing theorem. (1) $\Rightarrow(2)$ follows from the definition. $(2) \Rightarrow(3)$ : Since $\operatorname{Spec}(M)$ is strongly detectable by some Riemannian metric $h$ on $M$ with $|K(h)| \leq 1$, there exists, by definition, a positive number $\varepsilon$ such that $\lambda_{k}(M, h) \geq \varepsilon \lambda_{k}(M)$ for all $k \geq 1$. Thus

$$
Z(M, h ; t) \leq Z(M ; \varepsilon t) \leq Z(M, g ; \varepsilon t)
$$

for any Riemannian metric $g$ on $M$ with $|K(g)| \leq 1$. The MinakshisundaramPleijel asymptotic expansion applies for $h$ and $g$ to obtain

$$
\operatorname{vol}(M, h) \leq \varepsilon^{-n / 2} \operatorname{vol}(M, g) .
$$

This then gives $\operatorname{minvol}(M) \geq \varepsilon^{n / 2} \operatorname{vol}(M, h)>0$.

To see $(3) \Rightarrow(4)$, consider any Riemannian metric $g$ on $M$ with $|K(g)| \leq 1$. From the definition of the packing function $P(M, g ; \varepsilon)$ we have

$$
\operatorname{minvol}(M) \leq \operatorname{vol}(M, g) \leq \sum_{i=1}^{P(M, g ; \varepsilon)} \operatorname{vol}\left(B\left(x_{i}, 2 \varepsilon\right)\right)
$$

The Bishop volume comparison theorem ([BC]) implies that $\operatorname{vol}\left(B\left(x_{i}, \varepsilon\right)\right) \leq$ $\tilde{v}_{n}(2 \varepsilon)$ for all $i$ where $\tilde{v}_{n}(r)$ denotes the volume of a $r$-ball in the simply connected space $n$-form $M_{-1}^{n}$ with constant curvature -1 . Thus we obtain from (i) that

$$
P(M, g ; \varepsilon) \geq \frac{\operatorname{minvol}(M)}{\tilde{v}_{n}(2 \varepsilon)}
$$

for any Riemannian metric $g$ on $M$ with $|K(g)| \leq 1$. This gives

$$
P(M ; \varepsilon) \geq \frac{\operatorname{minvol}(M)}{\tilde{v}_{n}(2 \varepsilon)} .
$$

Since $\lim _{\varepsilon \rightarrow 0} \varepsilon^{-n} \tilde{v}_{n}(2 \varepsilon)=c_{3}(n)$ for some constant $c_{3}(n)$ depending only on $n$, there exists a constant $c_{4}(n)$ such that for any positive $\varepsilon \leq 1$,

$$
P(M ; \varepsilon) \geq \frac{\operatorname{minvol}(M)}{c_{4}(n) \varepsilon^{n}}>0 .
$$

On the other hand Theorem 2A implies that for any Riemannian metric $g$ on $M$ with $|K(g)| \leq 1$ we have

$$
\lambda_{P(M ; \varepsilon)}(M, g) \leq \lambda_{P(M, g ; \varepsilon)}(M, g) \leq \lambda(\varepsilon) .
$$


This gives

$$
\lambda_{P(M ; \varepsilon)}(M) \leq \lambda(\varepsilon) .
$$

For any integer $k \geq 1$ set $\varepsilon_{k}=\inf \{\varepsilon \mid P(M, \varepsilon) \leq k\}$. Choose a $k_{0}$ so that $\varepsilon_{k_{0}}<1$. Then we have from (iv) that

$$
k \geq P(M, \varepsilon) \geq \frac{\operatorname{minvol}(M)}{c_{4}(n) \varepsilon^{n}}
$$

for all $k \geq k_{0}$ and any $\varepsilon$ with $\varepsilon_{k}<\varepsilon \leq 1$. Thus one obtains that for $k \geq k_{0}$,

$$
k \geq \frac{\operatorname{minvol}(M)}{c_{4}(n) \varepsilon_{k}^{n}}
$$

From the fact that $\lim _{\varepsilon \rightarrow 0} \varepsilon^{2} \lambda(\varepsilon)=c_{5}(n)$, Inequalities (iv), (vi) and (viii) imply

$$
\begin{aligned}
\limsup _{k \rightarrow \infty} \lambda_{k}(M) k^{-2 / n} & \leq \limsup _{k \rightarrow \infty} \lambda_{P\left(M, \varepsilon_{k} / 2\right)}(M) k^{-2 / n} \\
& \leq \limsup _{k \rightarrow \infty} \varepsilon_{k}^{2} \lambda\left(\frac{\varepsilon_{k}}{2}\right)\left(\frac{c_{4}(n)}{\operatorname{minvol}(M)}\right)^{2 / n} \\
& =4 c_{5}(n)\left(\frac{c_{4}(n)}{\operatorname{minvol}(M)}\right)^{2 / n}<\infty
\end{aligned}
$$

and thus we have $(3) \Rightarrow(4)$.

Next we verify $(4) \Rightarrow(5)$. Suppose that $\operatorname{dim}_{s}(M)<n$, then the Weyl-type asymptotic formula applies for $\beta$ with $\operatorname{dim}_{s}(M)<\beta<n$ to obtain $\lambda_{k}(M) \geq$ $c_{\beta} k^{2 / \beta}$ for all $k \geq 1$ where $c_{\beta}$ is a positive constant. This contradicts with (4) and hence we have $\operatorname{dim}_{s}(M)=n$. From this, the statement (4) and the Weyl-type asympototic formula (or the Karamata's Tauberian theorem) imply easily that $s(M)>0$. To see $(5) \Rightarrow(1)$, one considers any Riemannian metric $g$ on $M$ with $|K(g)| \leq 1$. The Weyl asymptotic formula gives $\lambda_{k}(M, g) \sim c_{n} \operatorname{vol}(M, g)^{-2 / n} k^{2 / n}$. Thus we have

$$
\lim _{k \rightarrow \infty} \frac{\lambda_{k}(M, g)}{\lambda_{k}(M)}=\lim _{k \rightarrow \infty} \frac{c_{n} \operatorname{vol}(M, g)^{-2 / n} k^{2 / n}}{c(n) s(M)^{-2 / n} k^{2 / n}}=\frac{c_{n} \operatorname{vol}(M, g)^{-2 / n}}{c(n) s(M)^{-2 / n}}>0 .
$$

Hence one can find a positive number $\varepsilon$ such that $\lambda_{k}(M, g) \geq \varepsilon \lambda_{k}(M)$ for all $k \geq 1$. That is, $\operatorname{Spec}(M)$ is strongly detectable by $g$. The proof of the noncollapsing theorem is now completed. 
Remark. From this theorem one can conclude that $\operatorname{Spec}\left(S^{n}\right)$ is strongly detectable if and only if $n$ is even. This follows from the fact that $\operatorname{minvol}\left(S^{n}\right)>0$ if and only if $n$ is even. On the other hand the non-collapsing theorem also shows that if a two-surface $M$ has $\operatorname{dim}_{s}(M)<2$, then $M$ is either the 2 -torus $T^{2}$ or the Klein bottle $K^{2}$ and $\operatorname{dim}_{s}(M)=0$; compare with Proposition 3 .

So far we have handled the cases when the underlying manifold $M$ is totally collapsing or non-collapsing. For a partially collapsing manifold $M$ we shall investigate the relation between its spectral dimension $\operatorname{dim}_{s}(M)$ and collapsing dimension $\operatorname{dim}_{c}(M)$. First we show that the spectrum dimension is bounded below by the collapsing dimensin for a manifold with detectable topological spectrum.

The inverse collapsing theorem. If $\operatorname{Spec}(M)$ is detectable, one has $\operatorname{dim}_{c}(M) \leq \operatorname{dim}_{s}(M)$.

Proof. Since $\operatorname{Spec}(M)$ is detectable, there exists a sequence of Riemannian metric $g_{j}$ on $M$ with $|K(g)| \leq 1$ and a positive number $\varepsilon$ such that $\lim _{j \rightarrow \infty} \lambda_{k}\left(M, g_{j}\right) / \lambda_{k}(M) \geq \varepsilon$ for all $k \geq 1$. Using the standard diagonal argument, one can find a subsequence of Riemannian metrics, still denoted by $g_{j}$, such that

$$
\lambda_{k}\left(M, g_{j}\right) \geq \frac{\varepsilon}{2} \lambda_{k}(M)
$$

for all $k \geq 1$ and $j \geq k$. For any $\beta>\operatorname{dim}_{s}(M)$ the Weyl-type asymptotic formula gives

$$
\lambda_{k}(M) \geq c_{\beta} k^{2 / \beta}
$$

for all $k \geq 1$ where $c_{\beta}$ is a positive constant depending on $M$ and $\beta$. From (1) and (2) we have

$$
\lambda_{k}\left(M, g_{j}\right) \geq \frac{\varepsilon c_{\beta}}{2} k^{2 / \beta}
$$

for all $k \geq 1$ and $j \geq k$. Now fix a $k_{0}$ so that $\left(\varepsilon c_{\beta} / 2\right) k_{0}^{2 / \beta} \geq n^{2} / 4$. Theorem 2 implies that

$$
\frac{n^{2}}{4} \leq \lambda_{k_{0}}\left(M, g_{j}\right) \leq \lambda\left(\frac{\operatorname{diam}\left(M, g_{j}\right)}{2 k_{0}}\right)
$$

for all $j \geq k_{0}$. Hence $\operatorname{diam}\left(M, g_{j}\right) \leq 2 k_{0} \alpha_{n}$ for all $j \geq k_{0}$ where the constant $\alpha_{n}$ satisfies $\lambda\left(\alpha_{n}\right)=n^{2} / 4$ as in the proof of the lower bound theorem. 
It is now well-known that for any $D>0$ the class $\mathbb{M}(n, D)$ of all closed Riemannian $n$-manifolds $(M, g)$ with $K(g) \geq-1$ and $\operatorname{diam}(M, g) \leq D$ is precompact in the Hausdorff topology (cf. [G5]). Let $d_{j}$ denote the induced metric on $M$ by $g_{j}$. Without loss of generality we can assume that $\left(M, d_{j}\right)$ converges, in the Hausdorff topology, to a compact metric space $(X, d)$. To complete the proof, we need to verify that $\operatorname{dim} X \leq \beta$. To see this we shall use the packing functions $P(X, d ; \eta)$ and $P\left(M, g_{j} ; \eta\right)$ of $(X, d)$ and $\left(M, g_{j}\right)$ respectively. Since $\lim \left(M, d_{j}\right)=(X, d)$, there exists for any positive $\eta$ an integer $j(\eta)$ such that for any $j \geq j(\eta)$

$$
P(X, d ; \eta) \leq P\left(M, g_{j} ; \frac{\eta}{2}\right) \leq P\left(X, d ; \frac{\eta}{4}\right) .
$$

For any $j \geq \max \{P(X, d ; \eta / 4), j(\eta)\}$, Inequalities (3) and (5) imply

$$
\lambda_{P\left(M, g_{j} ; \eta / 2\right)}\left(M, g_{j}\right) \geq \frac{\varepsilon c_{\beta}}{2}\left(P\left(M, g_{j} ; \frac{\eta}{2}\right)\right)^{2 / \beta} \geq \frac{\varepsilon c_{\beta}}{2} P(X, d ; \eta)^{2 / \beta} .
$$

Theorem $2 \mathrm{~A}$ and the fact that $\lim _{\varepsilon \rightarrow 0} \varepsilon^{2} \lambda(\varepsilon)=c_{5}(n)$ imply that there exists a constant $c_{6}(n)$ such that

$$
\lambda_{P\left(M, g_{j} ; \eta / 2\right)}\left(M, g_{j}\right) \leq \lambda\left(\frac{\eta}{2}\right) \leq c_{6}(n) \eta^{-2}
$$

for any positive $\eta<1$. From this and (6) one obtains

$$
P(X, d ; \eta) \leq\left(\frac{2 c_{6}(n)}{\varepsilon c_{\beta}}\right)^{\beta / 2} \eta^{-\beta}
$$

for any positive $\eta<1$. The Pontrjagin-Schnirelman theorem ([PS], $[\mathrm{N}]$ ) then gives from (8) that $\operatorname{dim} X \leq \beta$. Thus $\operatorname{dim}_{c}(M) \leq \beta$. Since $\beta$ is an arbitrary number with $\beta>\operatorname{dim}_{s}(M)$, we conclude that $\operatorname{dim}_{c}(M) \leq \operatorname{dim}_{s}(M)$ and this completes the proof of the inverse collapsing theorem.

Now we can prove the topological structure theorem in the introduction.

Proof of the topological structure theorem. Let $M$ be a closed smooth $n$-manifold. The non-collapsing theorem implies that if the minimal volume of $M$ is non-zero, then it is strongly detectable. Thus, if $\operatorname{Spec}(M)$ is undetectable, one has $\operatorname{minvol}(M)=0$. This proves Part (1). Part (2) also follows easily from the non-collapsing thorem. To prove Part (3), we first observe that in the proof of the inverse collapsing theorem, one can find, for any $\beta>\operatorname{dim}_{s}(M)$, a compact metric space $X$ with $\operatorname{dim}(X) \leq \beta$ and a sequence of Riemannian metrics $g_{j}$ on $M$ with $\left|K\left(g_{j}\right)\right| \leq 1$ such that $\left(M, g_{j}\right)$ converges to $X$ in the Hausdorff topology. Since the dimension of $X$ is always an integer, we can find such a $X$ with $\operatorname{dim}(X) \leq \operatorname{dim}_{s}(M)$. In view of these properties, Part (3) follows directly from the following version of a compactification result of Fukaya. 
Theorem (Fukaya [F1], [F3]). Let $X$ be a compact metric space. Assume that a sequence of closed Riemannian n-manifolds $\left(M_{j}, g_{j}\right)$ with $\left|K\left(g_{j}\right)\right| \leq 1$ conveges to $X$ in the Hausdorff topology. Then we have the following:

(1) $X$ has a stratification $\varnothing=S_{\operatorname{dim}(X)+1}(X) \subset S_{\operatorname{dim}(X)}(X) \subset \cdots \subset S_{1}(X) \subset$ $S_{0}(X)=X$ such that $S_{k}(X)-S_{k+1}(X)$ is a smooth manifold of dimension $(\operatorname{dim}(X)-k)$;

(2) there is a differentiable map $f_{j}: M_{j} \rightarrow X$ such that the restriction of $f_{j}$ to $f_{j}^{-1}\left(S_{k}(X)-S_{k+1}(X)\right)$ is a fiber bundle whose fiber is diffeomorphic to an infranilmanifold.

(3) $X$ is isometric to the quotient space $N / O(n)$ of a smooth manifold $N$ with a $C^{1, \alpha}$-Riemannian metric by a smooth and isometric action of the orthogonal group $O(n)$. Moreover, the map $f_{j}: M_{j} \rightarrow X$ lifts to a map $\tilde{f}_{j}$ from the bundle of the orthogonal frames $F M$ of $M$ into $N$ such that $\tilde{f}_{j}$ is a fiber bundle with an infranilmanifold fiber.

From the inverse collapsing theorem, one may wonder when a manifold $M$ has $\operatorname{dim}_{s}(M) \leq \operatorname{dim}_{c}(M)$. So far, it is not clear to the author whether this is true in general. However, if the collapsing dimension $\operatorname{dim}_{c}(M)$ of $M$ is realized by a limit Riemannian manifold $N$, then this is true. To see this, we prove:

The direct collapsing theorem. If $M$ collapses to $N$, one has

$$
\operatorname{dim}_{s}(M) \leq \operatorname{dim}(N) .
$$

In case that $\operatorname{dim}_{s}(M)=\operatorname{dim}(N)>0$ and $s(M)>0$, then $\operatorname{Spec}(M)$ is detectable.

Proof. Our main tool to prove this theorem is based on some results of Fukaya ([Fu2]). If $N$ is a point, then $M$ is an infranilmanifold. Hence $\operatorname{dim}_{s}(M)=$ $\operatorname{dim}_{c}(M)=0$. Therefore, we can assume that $\operatorname{dim}(N) \geq 1$. Since $M$ collapses to $N$, there exist, by definition, a sequence of Riemannian metrics $g_{j}$ and $h$ on $M$ and $N$ respectively with $\left|K\left(g_{j}\right)\right| \leq 1$ such that $\lim \left(M, g_{j}\right)=(N, h)$ in the Hausdorff topology. Let $\Omega_{j}$ denote the volume form of $\left(M, g_{j}\right)$ and consider the measure $\mu_{j}=\left(1 / \operatorname{vol}\left(M, g_{j}\right)\right) \Omega_{j}$ on $\left(M, g_{j}\right)$. According to Theorems 0.4-0.8 in $[\mathrm{Fu} 2]$, we can conclude that $\left(M, g_{j}, \mu_{j}\right)$ converges to $(N, h, \mu)$ in the measured Hausdorff topology where $\mu$ is a measure on $N$ with the properties: $(1) \mu(N)=1$ and (2) $\mu=\chi \Omega_{N}$. Here $\chi$ is a continuous positive function on $N$ and $\Omega_{N}$ is the volume form of $(N, h)$. Moreover, the eigenvalues of the Laplace operator $\Delta$ of $\left(M, g_{j}\right)$ converge to those of a symmetric bilinear form $Q$ on $C^{\infty}(N)$ which is defined by

$$
Q(f, g)=\int_{N}\langle d f, d g\rangle d \mu
$$


for any $f$ and $g$ in $C^{\infty}(N)$. In other words, one has

$$
\lim _{j \rightarrow \infty} \lambda_{k}\left(M, g_{j}\right)=\lambda_{k}(Q) .
$$

To complete the proof of the direct collapsing theorem, we need to estimate the growth of the eigenvalues $\lambda_{k}(Q)$ of $Q$. Let $c_{1}$ and $c_{2}$ be the minimum and maximun values of the funcion $\chi$ on $N$. That is, for any $x \in N$,

$$
0<c_{1} \leq \chi(x) \leq c_{2} .
$$

Then the min-max principle gives

$$
\lambda_{k}(Q)=\inf _{\Lambda^{k}} \sup _{f \in \Lambda^{k}-\{0\}} \frac{\int_{N}\langle d f, d f\rangle d \mu}{\int_{N} f^{2} d \mu}
$$

where $\Lambda^{k}$ runs over all $k$-dimensional subspaces of $C^{\infty}(N)$. Hence $(2)$ and the min-max principle imply

$$
\lambda_{k}(Q) \geq \frac{c_{1}}{c_{2}} \inf _{\Lambda^{k}} \sup _{f \in \Lambda^{k}-\{0\}} \frac{\int_{N}\langle d f, d f\rangle \Omega_{N}}{\int_{N} f^{2} \Omega_{N}}=\frac{c_{1}}{c_{2}} \lambda_{k}(N, h) .
$$

From (1) and (4) we conclude that

$$
\lambda_{k}(M) \geq \frac{c_{1}}{c_{2}} \lambda_{k}(N, h)
$$

for all $k \geq 1$. The Weyl asymptotic formula applies for $(N, h)$ to obtain

$$
\lambda_{k}(M) \geq \frac{c_{1} c_{3}}{c_{2}} k^{2 / \operatorname{dim}(N)}
$$

for all $k \geq 1$ where $c_{3}$ is a positive constant depending only on $(N, h)$. Finally, the Weyl-type asymptotic formula for the topological spectrum implies that $\operatorname{dim}_{s}(M) \leq \operatorname{dim}(N)$.

In case that $\operatorname{dim}_{s}(M)=\operatorname{dim}(N)>0$ and $s(M)>0$, then the argument above and the Weyl-type asymptotic formula give that for any $k \geq 1$

$$
\lim _{j \rightarrow \infty} \lambda_{k}\left(M, g_{j}\right)=\lambda_{k}(Q) \geq \frac{c_{1} c_{3}}{c_{2}} k^{2 / m}
$$

and thus

$$
\lim _{j \rightarrow \infty} \frac{\lambda_{k}\left(M, g_{j}\right)}{\lambda_{k}(M)} \geq \frac{c_{1} c_{3} s(M)^{2 / m}}{c_{2} c(m)}>0
$$

where $m$ denotes the dimension of $N$. This implies that $\operatorname{Spec}(M)$ is detectable and the proof of the direct collapsing theorem is completed. 
We know that for a 2-manifold $M, \operatorname{Spec}(M)$ is either strongly detectable or $M$ is totally collapsing. As in Example 5, we can consider a solv-manifold Sol which is not an infranilmanifold such that Sol collapses to a circle $S^{1}$. For $n \geq 4$ such a solv-manifold, Sol, exists. See $[\mathrm{P}]$ for discussions of such manifolds. Then we have $\operatorname{dim}_{c}(\mathrm{Sol})=1$ and this is realized by a smooth Riemannian manifold $S^{1}$. Hence the direct collapsing theorem implies that $\operatorname{dim}_{s}(\mathrm{Sol}) \leq 1$. On the other hand, Proposition 2 gives that $\operatorname{dim}_{s}(\mathrm{Sol}) \geq 1$. Thus, one has $\operatorname{dim}_{s}(\mathrm{Sol})=1$. This example indicates the following:

Proposition 5. The degree of the growth of $R(n, k)$ in the gap theorem as $k$ goes to infinity is optimal for $n \neq 3$.

From the discussions in this section and Section 2, it is not too difficult to see that simply connected compact rank one symmetric spaces are strongly detectable; all infranilmanifolds are undetectable while the solv-manifold above, Sol, is detectable.

Next we discuss the topological spectrum of aspherical manifolds. Recall that a manifold $M$ is said to be aspherical if the homotopy groups $\pi_{k}(M)$ are trivial for all $k \geq 2$. In other words, its universal covering space is contractible. Typical examples of aspherical manifolds are nilmanifolds, solv-manifolds and locally symmetric spaces $\Gamma \backslash G / K$.

The compact limit space $(X, d)$ of an aspherical manifold $M$ has been investigated in [Fu3], [Fu4]. There Fukaya showed that if $\operatorname{dim}(X)>0, X$ is isometric to a quotient $Y / \Gamma$ where $Y$ is a smooth contractible manifold with a $C^{1, \alpha}$ Riemannian metric $g$ and $\Gamma$ is a properly discontinuous group of isometries of $Y$. In particular, $X$ is an orbifold. Note that the volume form $\Omega_{Y}$ and the Laplace operator $\Delta_{Y}$ induce canonically a volume form $\Omega_{X}$ and a Laplace operator $\Delta_{X}$ on $X$.

Now suppose that the limit space $(X, d)$ of an aspherical manifold $M$ realizes the collapsing dimension of $M$, i.e., $\operatorname{dim}_{c}(M)=\operatorname{dim}(X)$. Then we have a sequence of Riemannian metrics $g_{j}$ on $M$ with $\left|K\left(g_{j}\right)\right| \leq 1$ such that $\lim _{j \rightarrow \infty}\left(M, d_{j}\right)$ $=(X, d)$ in the Hausdorff topology where $d_{j}$ is the induced metric on $M$ by $g_{j}$. If $\operatorname{dim}(X)>0$, then $X$ is an orbifold. Thus Theorems 0.4-0.8 in [Fu2] imply again that $\left(M, d_{j}, \mu_{j}\right)$ converges to $(X, d, \mu)$ in the measured Hausdorff topology where $\mu_{j}=1 / \operatorname{vol}\left(M, g_{j}\right) \Omega_{\left(M, g_{j}\right)}$ and $\mu$ is a measure on $X$ with the properties: (1) $\mu(X)=1$ and (2) $\mu=\chi \Omega_{X}$. Here $\chi$ is a positive continuous function on $X$. Furthermore, the eigenvalues of the Laplace operator of $\left(M, g_{j}\right)$ converge to those of the associated symmetric bilinear form $Q$ on $C^{\infty}(X)$ of $\mu$ :

$$
Q(f, g)=\int_{X}\langle d f, d g\rangle d \mu
$$


for all $f$ and $g$ in $C^{\infty}(X)$. The argument in the proof of the direct collapsing theorem will then show that $\lambda_{k}(M)$ has the growth of degree at least $2 / \operatorname{dim}(X)$ if we have the Weyl asymptotic formula for the Laplace operator of $\left(X, \Omega_{X}\right)$. To see this, we can appeal to a result and its proof of Donnelly $([D])$. It states that the Weyl asymptotic formula is still valid for an orbifold which is the compact quotient of a non-compact Riemannian manifold by a properly discontinuous group of isometries acting on the manifold. In summary, the argument above yields:

Theorem 6. For an aspherical manifold $M$, one has $\operatorname{dim}_{s}(M) \leq \operatorname{dim}_{c}(M)$. In case that $\operatorname{dim}_{c}(M)=\operatorname{dim}_{s}(M)>0$ and $s(M)>0$, then $\operatorname{Spec}(M)$ is detectable.

From the discussions in Sections 2 and 3, it seems to the author that the spectral dimension of a smooth closed manifold behaves like its collapsing dimension. This leads to the following:

Conjecture. For a smooth closed manifold $M$, the spectral dimension, $\operatorname{dim}_{s}(M)$, is an integer and $\operatorname{dim}_{s}(M)=\operatorname{dim}_{c}(M)$.

In a forthcoming paper, we shall discuss this conjecture for manifolds with detectable topological spectra. Finally, let us conclude this note by the following proposition.

Proposition 6. Let $M$ and $N$ be two smooth closed manifolds with dimensions $\geq 2$. Then we have $\operatorname{dim}_{s}(M \times N) \leq \operatorname{dim}_{s}(M)+\operatorname{dim}_{s}(N)$.

Proof. Given any Riemannian metrics $g$ and $h$ on $M$ and $N$ respectively with $|K(g)| \leq 1$ and $|K(h)| \leq 1$, the product metric $g \times h$ on $M \times N$ also has $|K(g \times h)| \leq 1$. It is well known ([BGM]) that

$$
Z(M \times N, g \times h ; t)=Z(M, g ; t) Z(N, h ; t)
$$

for any $t>0$. This gives $Z(M \times N ; t) \leq Z(M ; t) Z(N ; t)$ for any $t>0$. Thus we have $\operatorname{dim}_{s}(M \times N) \leq \operatorname{dim}_{s}(M)+\operatorname{dim}_{s}(N)$ and the proposition holds.

Acknowledgment. The author would like to thank the referee for his comments and suggestions concerning this paper. 


\section{REFERENCES}

[BC] R. Bishop $\&$ R. L. CRittendon, Geometry of Manifolds, Academic Press, New York, 1974.

[Be] P. H. BERARD, Spectral geometry: Direct and Inverse Problems, Spring-Verlag, New York, 1986.

[Bu] P. BusER, Riemannsche Flächen mit Eigenwerte in (0, $\left.\frac{1}{4}\right)$, Comment. Math. Helv. 52 (1977), 25-34.

[BBG] P. Berard, G. Besson \& S. Gallot, Sur une inégalité isopérimétrique qui généralise celle de Paul-Lévy-Gromov, Invent. Math. 80 (1985), 295-308.

[BGM] M. Berger, P. GAuduchon \& E. MAZET, Le spectre dúne variété riemanniennes, Spring-Verlag, New York, 1971.

[BK] P. Buser \& H. KARCher, Astérisque 81 (1981), Gromov's almost flat manifolds.

[Ch] S. Y. CHENG, Eigenvalues comparison theorems and its geometric applications, Math. Z. 143 (1975), 289-297.

[Cl] I. ChaVel, Eigenvalues in Riemannian geometry, Academic Press, New York, 1984.

[D] H. Donnelly, Asymptotic expansions for the compact quotients of properly discontinuous group actions, Illinois J. Math. 23 (1979), 485-496.

[Fe] W. FELLER, An Introduction to the Theory of Probability, $2^{\text {nd }}$ ed., vol. II, Wiley, New York, 1971.

[Fu1] K. FUKAYA, Hausdorff convergnce of Riemannian manifolds and its applications, (Preprint).

[Fu2] Collapsing of Riemannian manifolds and eigenvalues of Laplace operator, Invent. Math. 87 (1987), 517-547.

[Fu3] $ـ$ A boundary of the set of the Riemannian manifolds with bounded curvatures and diameters, J. Differential Geom. 28 (1988), 1-21.

[Fu4] - A compactness of a set of aspherical Riemannian orbifolds, A Fete of Topology (Matsumoto, Mizutani and Morita, eds.), Academic Press, Boston, 1988, pp. 391413.

[G1] M. Gromov, Volume and bounded cohomology, Publ. Math. IHES 56 (1983), 213307.

[G2] Almost flat manifolds, J. Differ. Geom. 13 (1978), 231-241.

[G3] , Paul Levy's isoperimetric inequality, Pretirage IHES (1980).

[G4] Curvature, diameter and Betti numbers, Comment. Math. Helv. 56 (1981), 179-195.

[G5] _ Structure Métrique pour jes les Variétés Riemanniennes, Cedic/Fernand Nathan, Paris, 1981.

[H] J. HERSCH, Quatre propriétés isopérimétrique de membranes sphérique, C. R. Acad. Sci. Paris 270 (1970), 1645-1648.

[LY] P. LI \& S. T. YAU, A new conformal invariant and its applications to the Willmore conjecture and the first eigenvalue for compact surfaces, Invent, Math. 69 (1982), 269-291.

[M] H. P. MCKEAN, An upper bound for the spectrum of $\Delta$ on a manifold of negative curvature, J. Differential Geom. 4 (1970), 359-366.

[N] J. NAGATA, Modern Dimension Theory, Heldermann Verlag, Berlin, 1983.

[P] X.-W. PENG, Collapsing Riemannian manifolds to the circle, Math. Z. 202 (1989), 289-298. 
[PS] L. Pontruagin \& L. Schnirelmann, Sur une propriété métrique de la dimension, Ann. Math. II (1932), 152-162.

[R] E. RuH, Almost flat manifolds, J. Differential Geom. 17 (1982), 1-14.

[W1] J.-Y. WU, Hausdorff convergence and sphere theorems, Proc. of Symposia in Pure Math. UCLA 1990 (ed. by R. Greene and S. T. Yau), Vol. 54 (1993), Part 3, pp. 685-692.

[W2] _ On almost isospectral manifolds, Indiana Univ. Math. J. 39 (1990), 13731381.

[W3] - On the structure of almost nonnegatively curved manifolds, J. Differential Geom. 35 (1992), 385-397.

[YY] P. C. YANG \& S. T. YAU, Eigenvalues of the Laplacian of compact Riemannian surfaces and minimal submanifolds, Ann. Sci. Ecole Norm. Sup. Pisa 7 (1980), 55-63.

Research for this paper was partially supported by an N. S. C. grant NSC84-2121-M-194-003, Taiwan.

Department of Mathematics

National Chung Cheng University

Ming-Hsiung, Chia-Yi 621 Taiwan

E-MAIL: jywu@math.ccu.edu.tw

Received: October 5th, 1994; revised: March 23rd, 1995. 